\title{
Plasmodium malariae and Plasmodium ovale infections in the China-Myanmar border area
}

\author{
Peipei Li ${ }^{1}$, Zhenjun Zhao ${ }^{1}$, Hua Xing ${ }^{2}$, Wenli Li ${ }^{2}$, Xiaotong Zhu ${ }^{3}$, Yaming Cao ${ }^{3}$, Zhaoqing Yang ${ }^{4}$, \\ Jetsumon Sattabongkot ${ }^{5}$, Guiyun Yan ${ }^{6}$, Qi Fan ${ }^{7^{*}}$ and Liwang Cui ${ }^{7^{*}}$
}

\begin{abstract}
Background: The Greater Mekong Subregion is aiming to achieve regional malaria elimination by 2030. Though a shift in malaria parasite species predominance by Plasmodium vivax has been recently documented, the transmission of the two minor Plasmodium species, Plasmodium malariae and Plasmodium ovale spp., is poorly characterized in the region. This study aims to determine the prevalence of these minor species in the China-Myanmar border area and their genetic diversity.
\end{abstract}

Methods: Epidemiology study was conducted during passive case detection in hospitals and clinics in Myanmar and four counties in China along the China-Myanmar border. Cross-sectional surveys were conducted in villages and camps for internally displaced persons to determine the prevalence of malaria infections. Malaria infections were diagnosed initially by microscopy and later in the laboratory using nested PCR for the SSU rRNA genes. Plasmodium malariae and $P$. ovale infections were confirmed by sequencing the PCR products. The $P$. ovale subtypes were determined by sequencing the Pocytb, Pocox 1 and Pog3p genes. Parasite populations were evaluated by PCR amplification and sequencing of the MSP-1 genes. Antifolate sensitivity was assessed by sequencing the dhfr-ts and dhps genes from the P. malariae and P. ovale isolates.

Results: Analysis of 2701 blood samples collected from the China-Myanmar border by nested PCR targeting the parasite SSU rRNA genes identified 561 malaria cases, including 161 Plasmodium falciparum, 327 P. vivax, 66 P. falciparum/P. vivax mixed infections, 4 P. malariae and 3 P. ovale spp. P. vivax and P. falciparum accounted for $>60$ and $\sim 30 \%$ of all malaria cases, respectively. In comparison, the prevalence of $P$. malariae and $P$. ovale spp. was very low and only made up $\sim 1 \%$ of all PCR-positive cases. Nevertheless, these two species were often misidentified as $P$. vivax infections or completely missed by microscopy even among symptomatic patients. Phylogenetic analysis of the SSU rRNA, Pocytb, Pocox 1 and Pog3p genes confirmed that the three P. ovale spp. isolates belonged to the subtype P. ovale curtisi. Low-level genetic diversity was detected in the MSP-1, dhfr and dhps genes of these minor parasite species, potentially stemming from the low prevalence of these parasites preventing their mixing. Whereas most of the $d h f r$ and dhps positions equivalent to those conferring antifolate resistance in $P$. falciparum and $P$. vivax were wild type, a new mutation S113C corresponding to the S108 position in pfdhfr was identified in two P. ovale curtisi isolates.

Conclusions: The four human malaria parasite species all occurred sympatrically at the China-Myanmar border. While P. vivax has become the predominant species, the two minor parasite species also occurred at very low prevalence but were often misidentified or missed by conventional microscopy. These minor parasite species displayed low levels of polymorphisms in the msp-1, dhfr and dhps genes.

\footnotetext{
*Correspondence: qifan10001@163.com; luc2@psu.edu

1 Dalian Institute of Biotechnology, Dalian, Liaoning, China

${ }^{7}$ Department of Entomology, Pennsylvania State University, University Park, PA, USA

Full list of author information is available at the end of the article
} 
Keywords: Plasmodium falciparum, Plasmodium vivax, Plasmodium malariae, Plasmodium ovale, Prevalence, Genetic diversity, Molecular identification

\section{Background}

Malaria in humans is caused by four main Plasmodium species (Plasmodium falciparum, Plasmodium vivax, Plasmodium malariae and Plasmodium ovale) and the zoonotic parasite Plasmodium knowlesi, which is found in many Southeast Asian countries [1]. The most virulent species $P$. falciparum is also the most prevalent parasite in Africa, while $P$. vivax is the most widely distributed parasite outside of Africa [2]. Compared with these two species, $P$. malariae and $P$. ovale spp. are much less prevalent and significantly under-studied. Plasmodium malariae is more or less sympatric with $P$. falciparum in distribution, mainly found in sub-Saharan Africa and southwest Pacific $[3,4]$. In comparison, P. ovale spp. were thought to have a much more limited distribution and mostly found in Africa and some islands of the west Pacific [5]. Most published data showed that the prevalence of these two species has been underestimated apparently due to low parasitaemia, morphological resemblance with $P$. vivax, and occurrence as mixed infections with the major parasite species [6-10]. The advent of PCR-based molecular diagnosis method has revolutionized the detection of low-density Plasmodium infections [11]. Molecular genotyping also led to the division of $P$. ovale spp. into two distinct subspecies: $P$. ovale curtisi (Poc, the classic type) and P. ovale wallikeri (Pow, the variant type) [12]. The nuclear genome sequences further confirmed that Poc and Pow are genetically distinct but morphologically indistinguishable [13]. Further refinements and applications of the molecular methods have enabled enhanced detection of these parasites in molecular surveillance in endemic countries (e.g., [14-17]) as well as imported cases [18].

In the Greater Mekong Subregion (GMS) of Southeast Asia, which includes Cambodia, China, Laos, Myanmar, Thailand, and Vietnam, recent achievements in malaria control have motivated countries within this region to plan for regional malaria elimination by 2030. In this region, all five Plasmodium parasites infecting humans co-exist [19], and $P$. vivax and P. falciparum are the predominant parasite species. In contrast, other parasite species have been detected at much lower prevalence. Plasmodium malariae infections have been described in Cambodia [20-22], China [16, 23], Laos [19], Myanmar [24, 25], Thailand [11, 26-28], and Vietnam [19, 29, 30]. The detection of field isolates with variations in both morphology and the SSU rRNA gene suggests that $P$. malariae may exhibit high genetic diversity [23, 24]. Similarly, $P$. ovale spp. have been found in all nations of the GMS including Cambodia [21, 22], Laos [31, 32], Myanmar [16, 33, 34], Thailand [11, 26, 27], and Vietnam [30, 35]. Although a $P$. ovale-like case was described in 1941 in Yunnan [36], most, if not all, $P$. ovale spp. reported in China in recent years were imported, mainly from Africa $[37,38]$. Both the classic and variant types of $P$. ovale spp. were identified in autochthonous as well as imported cases [19, 37].

In the final stage of malaria elimination, new strategies tailored for rapid identification of new cases, prevention of local spread, and efficient management of malaria introduction are critical. Especially, malaria in the GMS is highly heterogeneous with transmission foci located along international borders [39]. Hence, malaria re-introduction as a result of cross-border human migration needs to be dealt with, and malaria elimination may require regional cooperative initiatives [40]. Routine malaria surveillance in endemic regions largely relies on microscopy of Giemsa-stained blood smears and rapid diagnostic tests (RDT). Given that $P$. malariae and $P$. ovale infections often present with a low parasitaemia and occur as mixed infections with $P$. falciparum and $P$. vivax [23,33], molecular methods of detection provide a more accurate estimate of malaria epidemiology. In this study, blood samples collected from the China-Myanmar border area were analysed for malaria parasites using a nested PCR method.

\section{Methods}

\section{Study sites}

This molecular epidemiology study was carried out in two study sites on both sides of the China-Myanmar border. In Myanmar, passive case detection (PCD) was performed in a hospital located in the Laiza Township and several nearby malaria clinics, Kachin State, between May 2011 and December 2012. In 2012, to obtain malaria prevalence information, cross-sectional surveys (CSS) were conducted in ten surrounding villages. In addition, active case detection (ACD) was also conducted through weekly (April-September) or biweekly (October-March) home visits to assess clinical malaria. From 2010, both ACD and PCD activities were started in three nearby camps for internally displaced people (IDP), which were newly established as a result of internal military strife. The Myanmar study site is located in one of 
the designated Special Zones [25], where malaria burden is particularly heavy and local public health infrastructure is poor. In China, PCD was performed in county and township hospitals of Tengchong, Yingjiang, Longchuan and Ruili counties, Yunnan Province in 2011-2012. Yunnan is the most malaria prevalent province in China and imported cases as cross-border migration made up a major part of malaria cases in Yunnan in recent years [38, 41].

\section{Malaria parasite samples}

In Kachin, 1106 blood samples were obtained during PCD activities from febrile patients attending the hospital and malaria clinics, and 638 were from participants of two CSS (Table 1). In addition, 854 samples were collected from participants in three nearby IDP camps in 2012. Among them, 366 were from febrile patients attending the malaria clinic, 411 were healthy people during the CSS, and 77 were febrile patients identified during weekly or bi-weekly ACD visits. Further, 103 samples were collected from malaria patients attending hospitals in the four counties of Yunnan in 2011-2012. During these surveys, written informed consent and assent for minors were obtained and demographic data were collected using questionnaires. All diagnosis was done by microscopic examination of both thick and thin blood smears. All samples collected from Yunnan Province were evaluated by RDT (Malaria Pv/Pf Test Device, Tycolpharm Co., Limited, UK), a Pf/Pan device based on pan-pLDH [34]. Finger-prick blood drops were collected by trained nurses on Whatman $3 \mathrm{M}$ filter paper, air-dried, and stored at $-20{ }^{\circ} \mathrm{C}$ before processing. Parasite density was estimated using thick smears assuming 8000 leukocytes/ $\mu \mathrm{L}$ of blood [34]. The study protocol was reviewed and approved by the Institutional Review Boards of the Pennsylvania State University, USA, Kunming Medical University, China and the local Bureau of Health, Kachin State, Myanmar.

\section{Screening for Plasmodium malariae and Plasmodium ovale} spp

Genomic DNA was extracted from dried blood spots on filter paper using the Qiagen DNA Mini Kit according to the manufacturer's instruction (Qiagen, Germany). Nested PCR was performed to screen malaria infectious from these 2701 blood samples. Primers rPLU5 and rPLU6 were used for the primary PCR, whereas primers rFAL1/2, Pv18SF/R, Pm18SF/R, Po18SF/R, and Pk18SF/R, specific for each of the four human malaria parasite species as well as $P$. knowlesi, were used for the nested PCR [42]. The PCR positive samples of $P$. malariae and $P$. ovale spp. were further confirmed by sequencing the PCR products. Some of the blood smears with inconsistent diagnosis results between the original microscopy and PCR were reexamined to verify the presence of parasite-infected red blood cells.

\section{Molecular characterization of $P$. malariae and $P$. ovale isolates}

To further characterize the $P$. malariae and $P$. ovale isolates, several genes were amplified and sequenced using primers listed in Additional file 1. These include the cytochrome $b$ (cytb), cytochrome oxidase subunit 1 (cox1) and glyceraldehyde-3-phosphate $(g 3 p)$ genes of $P$. ovale spp., dihydropoteroate synthase (dhps) of $P$. malariae [43], dihydrofolate reductase (dhfr) and MSP-1 genes in both species [44-46]. Amplified fragments of DNA were purified, and sequenced directly or cloned into pMD18-T vector (Takara) for sequencing. Sequences were assembled using Lasergene (DNASTAR). The sequences determined in this study were deposited in GenBank with accession numbers KX672017-KX672048.

\section{Sequence analysis}

Sequences were searched in the GenBank by using the BLAST program. Orthologous sequences of $P$. malariae

Table 1 Surveillance of malaria in Kachin State, Myanmar and four border counties of Yunnan Province, China

\begin{tabular}{|c|c|c|c|c|c|c|c|c|c|}
\hline Location & Surveys & Method & Pf & Pv & Pm & Po & Pf and Pv & Total positive & Total \\
\hline \multirow[t]{6}{*}{ Myanmar } & PCD & Microscopy & 128 & 253 & 1 & 0 & 34 & $416(28.3 \%)$ & 1472 \\
\hline & & PCR & 136 & 257 & 3 & 2 & 63 & 461 (31.3\%) & \\
\hline & CSS & Microscopy & 4 & 13 & 0 & 0 & 3 & $20(1.9 \%)$ & 1049 \\
\hline & & PCR & 8 & 22 & 0 & 0 & 0 & $30(2.9 \%)$ & \\
\hline & $A C D$ & Microscopy & 0 & 1 & 0 & 0 & 0 & $1(1.3 \%)$ & 77 \\
\hline & & PCR & 0 & 1 & 0 & 0 & 0 & $1(1.3 \%)$ & \\
\hline \multirow[t]{2}{*}{ Yunnan } & PCD & RDT & 18 & 42 & 0 & 0 & 1 & $61(59.2 \%)$ & 103 \\
\hline & & PCR & 17 & 47 & 1 & 1 & 3 & 69 (67.0\%) & \\
\hline
\end{tabular}

Plasmodium species: Pf (P. falciparum), Pv (P. vivax), Pm (P. malariae), and Po (P. ovale)

$P C D$ passive case detection; $C S S$ cross-sectional surveillance; $A C D$ active case detection; $R D T$ rapid diagnostic test 
and $P$. ovale spp. were retrieved from the GenBank and PlasmoDB (http://www.plasmodb.org) for phylogenetic analysis. GenBank accession numbers of the genes included in sequence analysis are shown in Additional file 2. Nucleotide and deduced amino acid sequences were aligned by using CLUSTALW. Phylogenetic trees were constructed using the Neighbor-Joining method implemented in MEGA6 [47].

\section{Results}

Malaria case detection under the PCD and ACD efforts

For malaria surveillance along the China-Myanmar border, PCD was conducted in clinics and hospitals, while $A C D$ and CSS were performed in the surrounding villages and IDP camps near Laiza township of Kachin State, Myanmar. On the Myanmar side, malaria diagnosis in hospitals and clinics was performed primarily by microscopy, and overall $28.3 \%$ of patients with febrile illness were diagnosed as having malaria infections and treated (Table 1). In Kachin State, the ACD efforts detected only one malaria case from 77 (1.3\%) villagers with fever symptoms. Malaria prevalence in the villages and camps was low; CSS only detected $1.9 \%$ of the participants as having asymptomatic Plasmodium infections (Table 1). More specifically, malaria prevalence (slide positivity) in the villages was $1.3 \%(8 / 638)$, whereas it was slightly higher $(2.9 \%, 12 / 411)$ in the IDP camps. In this border region, $P$. vivax and $P$. falciparum were the predominant parasite species. In Myanmar, among the 437 malaria cases identified by microscopy, $P$. vivax, $P$. falciparum and $P$. malariae infections accounted for 61.1, 30.2 and $0.2 \%$, respectively (Table 1 ). In addition, $8.5 \%$ of these cases were diagnosed as mixed $P$. falciparum/P. vivax infections. In the four border counties of Yunnan Province, China, 59.2\% of febrile cases suspected for malaria infections were diagnosed by an RDT as malaria cases. Among the 61 positive cases, 68.9, 29.5 and 1.6\% were due to $P$. vivax, $P$. falciparum and mixed infections of these two species.

For a more accurate identification of parasite species, nested PCR targeting the parasite SSU rRNA genes was used to analyze the 2701 blood samples. For both PCD and CSS efforts, PCR detected more malaria cases (Table 1). For PCD in Myanmar, microscopy missed 9.8\% $(45 / 461)$ of malaria cases among the febrile patients. Furthermore, PCR detected $13.7 \%$ (63/461) of the malaria cases as mixed $P$. falciparum/P. vivax infection as compared to $7.4 \%$ (34/461) determined by microscopy. For the CSS efforts, PCR detected $2.9 \%$ of participants carrying Plasmodium infections, an increase of $1 \%$ compared with that of microscopic diagnosis. In Yunnan, PCR also improved the RDT diagnosis sensitivity by $7.8 \%$. In comparison, there were 41 discrepancies between the results of microscopy and PCR (Additional file 3). Six were slidepositive but PCR negative (one $P$. falciparum and five $P$. vivax). Of 26 cases diagnosed as mixed-species infections by microscopy, 22 were $P$. falciparum and 4 were $P$. vivax single infections. In addition, nine slide-positive cases were identified as infections by a different species (Additional file 3).

For the minor Plasmodium species, whereas no $P$. knowlesi infections were identified, PCR detected a total of four $P$. malariae and three $P$. ovale cases (Table 1). Of the four $P$. malariae cases, only one was correctly identified, and the remaining three were misdiagnosed as $P$. vivax infections by microscopy. In addition, one $P$. malariae case was diagnosed by the RDT as a $P$. vivax infection. Two $P$. ovale cases were missed by both microscopy and RDT, probably due to low parasitemias, whereas one was misdiagnosed as $P$. vivax infection. Both parasite species had very low prevalence of $1.2 \%(7 / 561)$ in PCR-confirmed positive cases. In total, infections by the two minor Plasmodium species only made up $0.26 \%$ (7/2701) of all febrile cases at the hospitals and clinics. It is also noteworthy that all $P$. malariae and $P$. ovale cases were from PCD surveys. Most $P$. malariae and $P$. ovale patients presented with fever at the time of examination $\left(>37.5{ }^{\circ} \mathrm{C}\right.$ ) and had $2-5$ days of fever histories (Table 2). These cases with disparate diagnosis results between the original microscopy and PCR were re-examined by an expert microscopist, who confirmed the PCR results.

\section{Confirmation of $P$. malariae and $P$. ovale cases}

The PCR products of the SSU rRNA genes of the two minor species from the positive cases were cloned and sequenced. BLAST analysis and alignment of sequences showed that the four $P$. malariae SSU rRNA gene sequences had $99 \%$ identities with those from published $P$. malariae sequences, whereas the $P$. ovale SSU rRNA gene sequences from the three isolates were most closely related to those of $P$. ovale spp. with $99 \%$ identities. Consistently, phylogenetic analysis showed all four $P$. malariae SSU rRNA gene sequences were grouped together with other $P$. malariae sequences, whereas the three clinical $P$. ovale isolates were clustered with $P$. ovale curtis $i$ (Fig. 1).

\section{Plasmodium ovale subtype characterization}

Plasmodium ovale spp. are divided into two genetically distinct subspecies, Poc and Pow [12]. To further confirm that the $P$. ovale isolates identified in this study belong to Poc, two mitochondrial genes Pocytb (508 bp) and Pocox 1 (861 bp), and one nuclear gene Pog3p (359 bp) from the three clinical isolates we amplified, sequenced and compared with the reference sequences (Table 3). It is obvious that the three clinical isolates were more closely 
Table 2 Characteristics of the $P$. malariae and $P$. ovale cases

\begin{tabular}{|c|c|c|c|c|c|c|c|c|}
\hline \multirow[t]{2}{*}{ Case number } & \multirow[t]{2}{*}{ Age } & \multirow[t]{2}{*}{ Sex } & \multirow[t]{2}{*}{ Microscopy } & \multirow[t]{2}{*}{ RDT } & \multirow[t]{2}{*}{ PCR } & \multirow{2}{*}{$\begin{array}{l}\text { Axillary } \\
\text { temperature }\end{array}$} & \multicolumn{2}{|c|}{ Parasite densityc } \\
\hline & & & & & & & Asexual & Gametocytes \\
\hline$M 0500214^{a}$ & 27 & $M$ & - & - & P. ovale & 40.0 & - & - \\
\hline M0102751 & 7 & $M$ & - & - & P. ovale & 38.6 & - & - \\
\hline$C 0100511^{b}$ & 33 & $M$ & $P \vee$ & PV & P. ovale & 38.9 & 800 & 400 \\
\hline MON00648 & 30 & $M$ & $\mathrm{Pm}$ & ND & P. malariae & 38.0 & 880 & 40 \\
\hline MON00556 & 11 & $\mathrm{~F}$ & $P \vee$ & ND & P. malariae & 39.5 & 2120 & 4800 \\
\hline MON00290a & 29 & $M$ & $P \vee$ & ND & P. malariae & 36.0 & 80 & 1600 \\
\hline $\mathrm{C} 0400117^{b}$ & 22 & $M$ & PV & PV & P. malariae & 40.5 & 400 & 160 \\
\hline
\end{tabular}

ND not done

a Cases detected in Myanmar

b Cases detected in Yunnan

c Parasites/ $\mu$ l of blood

related to Poc than to Pow. Phylogenetic analysis of these three genes all confirmed that the three $P$. ovale isolates belonged to the Poc subtype.

\section{Diversity of the MSP-1 genes}

To study the genetic diversity of these two minor Plasmodium species in the China-Myanmar border area, four overlapping fragments of the Pmmsp-1 gene and three overlapping fragments of the Pomsp-1 gene were amplified by nested PCR from three (3/4) P. malariae isolates (M0N00290, M0N00556 and M0N00648) and two (2/3) $P$. ovale isolates (C0100511 and M0500214), and sequenced. The assembled PmMSP-1 sequences were 5160 bp (M0N00648) and 5150 bp (M0N00290 and M0N00556) in length, whereas the PoMSP-1 sequences were both $5157 \mathrm{bp}$. Alignment of the PmMSP-1 sequences with the full-length sequence of a Cameroonian isolate MM1A (GenBank \#FJ824669) [45] revealed nucleotide and amino acid identities of $\sim 98$ and $\sim 97 \%$, respectively. Alignment of the PmMSP-1 sequences from this study with the sequences of 11 Brazilian isolates by fragments showed that fragment 2 sequences corresponding to amino acids $637-858$ in the MM1A sequence were the most polymorphic with $87.3-88.9$ and $82.3-83.6 \%$ identities in the nucleotide and amino acid sequences, respectively (Additional file 4). Fragment 4, corresponding to amino acids $1366-1512$ in the MM1A sequence, was absolutely conserved in the $P$. malariae isolates analyzed in this study.

The two Pomsp-1 sequences encoding 1718 amino acids were $100 \%$ identical. They were $>98 \%$ identical in nucleotide sequence with the Pocmsp-1 sequence from the Cameroonian isolates OM1A and OM1B (GenBank \#FJ824670 and FJ824671), and $91 \%$ identical with the Powmsp-1 from the Thai isolates (Additional file 5). Comparison of the amino acid sequences showed a similar trend. Compared with the PoMSP-1 gene from the Thai isolate Po-7 (GenBank No. KC137346) [48], there are two synonymous mutations (C421T and T1929C) and one nonsynonymous mutation $(\mathrm{C} 541 \mathrm{~T})$ in the isolate C0100511. A 12-nucleotide repeat sequence (GCCGCTACACAA) encoding amino acids AATQ between the position 2814-2911 in the variable domain 5 was found to have repeated three times in the Thai isolate and four times in both C0100511 and M0500214 (Additional file 6). The highest amino acid identity in the other variable domains and interspecies conserved sequences of PoMSP-1 was observed among the M0500214, C0100511 and five Poc isolates (Po-4, Po-7, Po-8, Po-9, Po-10) from Thailand.

\section{The $d$ hfr-ts genes from $P$. malariae and $P$. ovale isolates}

The dhfr-ts gene from the four P. malariae isolates and three $P O C$ isolates were amplified and sequenced. The four $P$. malariae dhfr-ts genes ranged in size from 1678 to $1779 \mathrm{bp}$, encoding proteins of 559-592 amino acids and corresponding to amino acids 13-604 of the PmDHFR-TS from the Thai isolate Pm3 (GenBank \#EF188271) [44] (Additional file 7). Three of the four isolates from the China-Myanmar border area had a PmDHFR sequence identical to that of the Thai Pm3 isolate, whereas M0N00648 contained two new mutations, L137P and K161R (Table 4; Additional file 7). All four PmDHFR-TS sequences from the China-Myanmar border isolates were wild type at amino acids S49, N50, S58, S114 and I170, which correspond to amino acids C50, N51, C59, S108 and I164 in PfDHFR, respectively. Mutations at these sites are known to confer pyrimethamine resistance $[49,50]$. Two of the four PmDHFR sequences also contained amino acids A15 corresponding to A16 in PfDHFR. Of note, the S49 and S58 in PmDHFR-TS were different from the C50 and C59 in the PfDHFR 


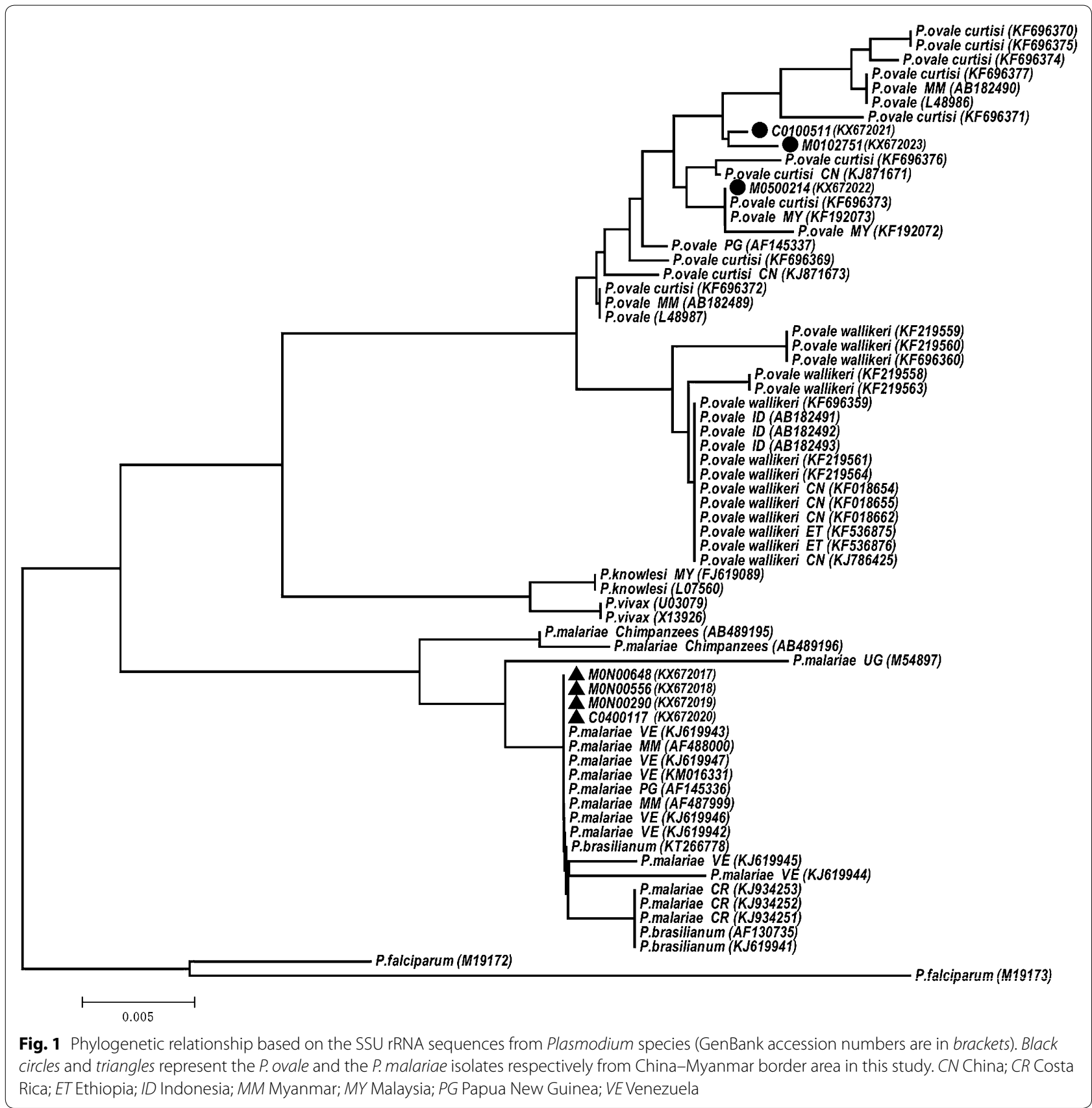

sequence. Mutations N50K, K55E, S58R, S59A, S114N/G and I170M found in other PmDHFR sequences $[44,51]$ were not observed in the PmDHFR sequences from the China-Myanmar border isolates (Table 4; Additional file 7).

The three sequences of the Podhfr-ts gene ranged from 1673 to $1757 \mathrm{bp}$ and encode proteins of 557-585 amino acids, corresponding to amino acids $24-608$ of the PocDHFR-TS from the Indian isolate Po2003 (GenBank
\#KP050405) [52] (Additional file 7). Sequence polymorphisms were found at positions A62, C113, H231 and E370 in PocDHFR-TS from the isolate M0102751, while isolate M0500214 only contained C113 (Table 4; Additional file 7). Similarly, Y49 and S58 in PocDHFR-TS were different from C50 and C59 in the PfDHFR-TS sequence. Also, C113 in PocDHFR-TS from the isolate M0102751 and M0500214 was different from S108 in the PfDHFRTS (Table 4). 
Table 3 Nucleotide substitutions in Pocyt b, Pocox 1 and Pog3p compared with the sequences in Poc and Pow

\begin{tabular}{|c|c|c|c|c|c|c|c|c|c|c|c|c|c|c|c|c|c|c|c|c|c|c|c|c|c|c|c|c|c|c|c|c|c|c|}
\hline \multirow[t]{3}{*}{ Isolates } & \multicolumn{34}{|c|}{ Genes } \\
\hline & \multicolumn{10}{|c|}{ Pocyt b (508 bp) } & \multicolumn{11}{|c|}{ Pocox 1 (861 bp) } & \multicolumn{13}{|c|}{$\boldsymbol{P o g} 3 p(359$ bp) } \\
\hline & 53 & 94 & 951 & 147 & 305 & 317 & 335 & 437 & 446 & 464 & 80 & 89 & 931 & 104 & 206 & 263 & 88 & 61 & 475 & 597 & 713 & 33 & 51 & 57 & 78 & 87 & 132 & 180 & 240 & 252 & 276 & 6285 & 5304 & 321 \\
\hline C0100511 & $T$ & $\mathbf{T}$ & $\mathrm{C}$ & A & G & $\mathrm{C}$ & $\mathbf{T}$ & $\mathbf{T}$ & C & $\mathrm{C}$ & $\mathbf{T}$ & $\mathbf{T}$ & $\mathrm{C}$ & $\mathrm{C}$ & $\mathbf{T}$ & $G$ & $\mathrm{C}$ & $\mathrm{C}$ & A & A & $\mathbf{T}$ & $\mathbf{T}$ & $\mathrm{C}$ & A & A & $\mathrm{T}$ & A & $\mathrm{C}$ & $\mathbf{A}$ & $\mathbf{T}$ & $\mathbf{T}$ & $\mathbf{T}$ & A & G \\
\hline M0102751 & $\mathbf{T}$ & A & $\mathrm{C}$ & A & G & $\mathrm{C}$ & $T$ & $\mathbf{T}$ & C & $\mathrm{C}$ & $T$ & $\mathbf{T}$ & $\mathrm{C}$ & $\mathrm{C}$ & $\mathbf{T}$ & G & $\mathrm{C}$ & $\mathrm{C}$ & $T$ & $\mathbf{A}$ & $\mathbf{T}$ & $\mathbf{T}$ & $\mathrm{C}$ & A & A & $T$ & A & $\mathrm{C}$ & $\mathbf{A}$ & $\mathbf{T}$ & $\mathbf{T}$ & $\mathbf{T}$ & $T$ & G \\
\hline M0500214 & $T$ & $\mathbf{T}$ & $\mathrm{C}$ & $\mathrm{G}$ & G & $\mathrm{C}$ & $T$ & c & C & $\mathrm{C}$ & $T$ & $\mathbf{T}$ & $\mathrm{C}$ & C & $T$ & $G$ & $\mathrm{C}$ & C & A & A & $\mathbf{T}$ & $\mathbf{T}$ & $\mathrm{C}$ & A & A & $T$ & A & $\mathrm{C}$ & A & $\mathbf{T}$ & $\mathbf{T}$ & $\mathbf{T}$ & A & G \\
\hline$P o c$ & $\mathbf{T}$ & $\mathbf{T}$ & $\mathrm{C}$ & A & G & $\mathrm{C}$ & $\mathbf{T}$ & $\mathbf{T}$ & C & $\mathrm{C}$ & $\mathbf{T}$ & $\mathbf{T}$ & $\mathrm{C}$ & $\mathrm{C}$ & $\mathbf{T}$ & $G$ & $\mathrm{C}$ & $\mathrm{C}$ & A & $\mathbf{A}$ & $\mathbf{T}$ & $\mathbf{T}$ & $\mathrm{C}$ & A & $\mathbf{A}$ & $\mathbf{T}$ & $\mathbf{A}$ & C & $\mathbf{A}$ & $\mathbf{T}$ & $\mathbf{T}$ & $\mathbf{T}$ & A & G \\
\hline Pow & A & $\mathbf{T}$ & $\mathbf{T}$ & A & $\mathbf{T}$ & $\mathbf{T}$ & A & $\mathbf{T}$ & $\mathbf{T}$ & $\mathbf{T}$ & $\mathrm{C}$ & $\mathrm{C}$ & $\mathbf{T}$ & $\mathbf{T}$ & G & $\mathbf{A}$ & $\mathbf{T}$ & $T$ & $\mathbf{A}$ & $G$ & $\mathbf{A}$ & A & $\mathbf{T}$ & $T$ & $\mathrm{G}$ & G & $T$ & $\mathbf{T}$ & C & $\mathrm{C}$ & C & A & A & $T$ \\
\hline
\end{tabular}

Numbering was according to the submitted sequence to GenBank. The GenBank access numbers: Pocyt b: KX672027 (M0500214), KX672028 (M0102751), KX672029 (C0100511); Pocox 1: KX672024 (M0500214), KX672025 (M0102751), KX672026 (C0100511); Pog3p: KX672030 (M0500214), KX672031 (M0102751), KX672032 (C0100511). The GenBank access numbers of the reference sequences for the three Poc genes are HQ712052, HQ712052 and KP050383, respectively; and for the three Pow genes are KP050428, KP050417, and KP050384, respectively. Nucleotides that are different from the type Poc sequence are shadowed

\section{Sequencing analysis of the dhps genes from P. malariae}

The Pmdhps gene was successfully amplified from the four P. malariae isolates. The partial Pmdhps gene was 962 bp in length and encoded 320 amino acids, corresponding to amino acids 347-659 in PfDHPS. The four PmDHPS sequences from the China-Myanmar border area isolates were identical to the PmDHPS haplotype 1 reported earlier [43] (Additional file 8). The six amino acids in PmDHPS equivalent to the residues associated with sulfadoxine resistance in PfDHPS (S436, K540, A581, I588 and A613) were all wild type except the mutant A437G.

\section{Discussion}

Accurate knowledge of the malaria epidemiology is essential for guiding control and elimination efforts. This study employed both ACD and PCD along the international borders between China and Myanmar, and used a molecular detection method in order to more accurately determine the infection prevalence among febrile patients and asymptomatic residents, and parasite species compositions. In this border region, malaria is seasonal with all four human malaria parasites. While $P$. vivax has become the predominant parasite species followed by $P$. falciparum $[16,53]$, the two minor parasite species $P$. malariae and $P$. ovale spp. were detected at very low frequencies in patients presenting in hospitals and clinics with acute febrile illness. It is important to note that three of the four P. malariae cases and one $P$. ovale case were misidentified as $P$. vivax infections by microscopy and treated with the chloroquine/primaquine combination, whereas two $P$. ovale cases were missed by microscopy and thus were not treated with anti-malarial drugs. Furthermore, PCR also identified more mixed-species infections, which may not have been treated properly depending on the results of the initial diagnosis. Thus, more sensitive diagnosis may well be needed for "border malaria" in order to completely eliminate malaria transmission.

Published data to date showed that the $d h f r$ genes in Poc and Pow were highly conserved in codons potentially mediating pyrimethamine resistance, although isolates carrying double A15S and S58R mutations were observed in imported cases in Singapore [12, 52]. The four $P$. malariae cases and three $P$. ovale cases detected in this study did not show mutations at equivalent residues responsible for antifolate resistance in other Plasmodium, while two new mutations at positions L137P and K161R were observed in one $P$. malariae isolate and four new mutations were observed at positions T62A, S113C, Y231H and K370E in Podhfr. It is noteworthy that the S113C mutation in the Pocdhfr corresponds to amino acid S108 in Pfdhfr, the primary position associated with pyrimethamine resistance $[50,54]$, but it is not known whether S113C mutation also confers pyrimethamine resistance in Poc. Similarly, Pmdhps gene also showed limited genetic diversity and all four isolates from the China-Myanmar border were identical to the PmDHPS haplotype 1, which was most prevalent in Asian countries [43].

MSP-1 as a predominant merozoite surface antigen is present in all examined Plasmodium species. P. falciparum and $P$. vivax MSP-1 genes display extensive genetic diversity, which is often used as a molecular marker for population studies. To date, limited studies on PmMSP-1 among Brazilian isolates and PocMSP-1 on Thai isolates detected low-level sequence diversity with most of variations located at interspecies variable domains of this gene [46, 48]. Phylogenetic analysis of PmMSP-1 using the maximum likelihood method placed the China-Myanmar border isolates separated from the Brazilian isolates, indicating divergent parasite populations. Furthermore, the PocMSP-1 sequences from the China-Myanmar border isolates were highly conserved and also displayed a 


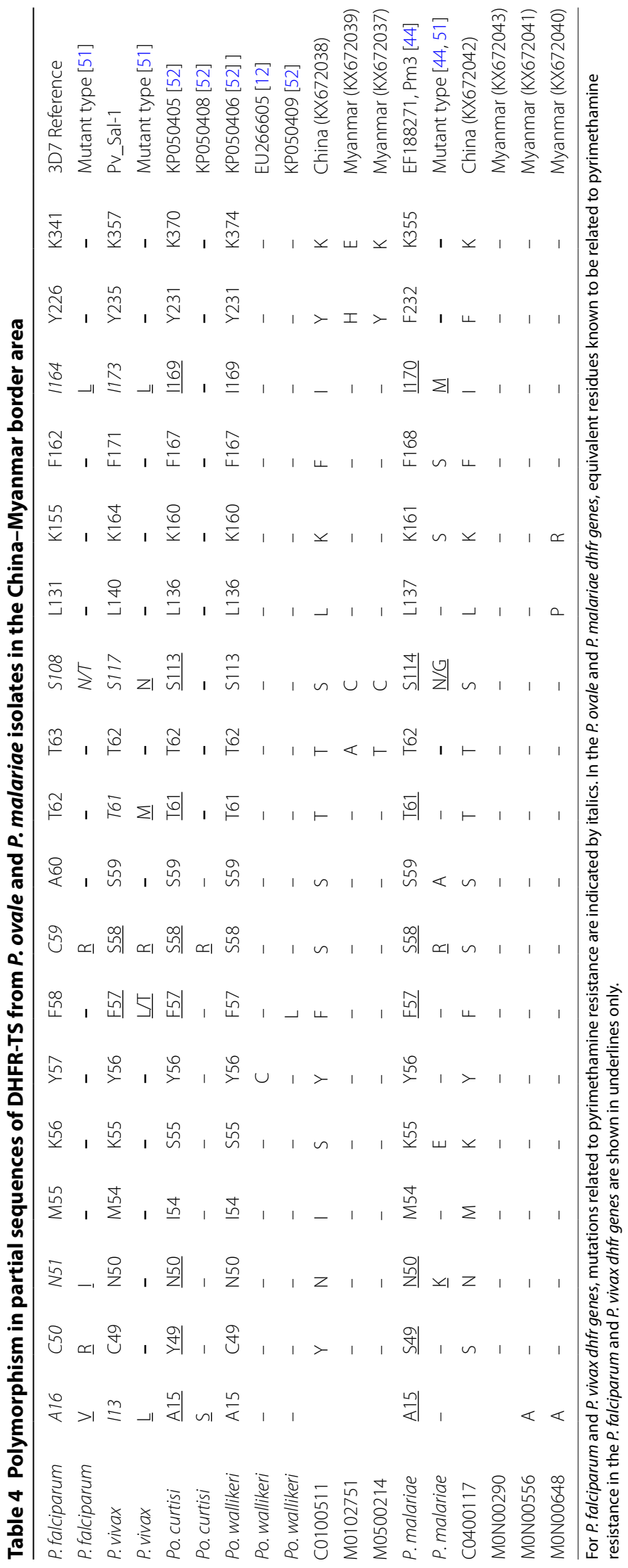


high level of similarity with the PocMSP-1 from the Thai isolates. Apparently, future studies with larger populations of the two minor parasite species are warranted.

\section{Conclusions}

Malaria epidemiology at the China-Myanmar border has shifted to $P$. vivax predominance, while the two minor parasite species $P$. malariae and $P$. ovale spp. also occurred at very low prevalence. However, these minor species were most often misidentified or missed, highlighting a potential problem in malaria treatment. Molecular studies identified the $P$. ovale spp. as the subspecies Poc. Characterization of two antifolate genes $d h f r$ and dhps revealed significant conservation at most positions possibly conferring antifolate resistance in $P$. falciparum and $P$. vivax, suggesting reduced impacts of antifolate selection on these two minor parasite species. Despite this, the S113C mutation, corresponding to S108 position in Pfdhfr, was first reported here in two Poc isolates from the China-Myanmar border area. Further analysis of the MSP-1 gene also revealed much lower levels of genetic diversity than their orthologs in P. falciparum and $P$. vivax populations, possibly due to the persistent low prevalence of these minor species in the GMS.

\section{Additional files}

Additional file 1. PCR primer sequences and reaction conditions.

Additional file 2. GenBank accession number of the orthologous sequences.

Additional file 3. Discrepancies in diagnosis between microscopy and PCR.

Additional file 4. Sequence identity between the PMMSP-1 sequences.

Additional file 5. Sequence identity between the POMSP-1 sequences.

Additional file 6. Alignment of PoMSP1 sequences.

Additional file 7. Sequence alignment of PmDHFR-TS (A) and PoDHFR-TS (B).

Additional file 8. Sequence alignment of PmDHPS.

\section{Abbreviations}

GMS: Greater Mekong Subregion; PCD: passive case detection; CSS: crosssectional surveys; ACD: active case detection; IDP: internally displaced people; RDT: rapid diagnostic tests; $m s p-1$ : merozoite surface protein-1; cytb: cytochrome b; cox1: cytochrome oxidase subunit 1; 93p: glyceraldehyde-3-phosphate; dhps: dihydropoteroate synthase; dhfr: dihydrofolate reductase.

\section{Authors' contributions}

$\mathrm{PL}, \mathrm{ZZ}, \mathrm{HX}$ and $\mathrm{XZ}$ carried out the experimental work and data analysis. WL, YW, ZY, JS and GY participated in data analysis. PL performed manuscript writing. QF and LC conceived the study and participated in writing. All authors read and approved the final manuscript.

\section{Author details}

${ }^{1}$ Dalian Institute of Biotechnology, Dalian, Liaoning, China. ${ }^{2}$ Dalian University of Technology, Dalian, Liaoning, China. ${ }^{3}$ Department of Immunology, China Medical University, Shenyang, Liaoning, China. ${ }^{4}$ Department of Parasitology, Kunming Medical University, Kunming, Yunnan, China. ${ }^{5}$ Faculty of Tropical
Medicine, Mahidol University, Bangkok 10400, Thailand. ${ }^{6}$ University of California, Irvine, CA, USA. ${ }^{7}$ Department of Entomology, Pennsylvania State University, University Park, PA, USA.

\section{Acknowledgements}

The authors thank the reviewers for their constructive comments and suggestions.

\section{Competing interests}

The authors declare that they have no competing interests.

\section{Availability of data and material}

All the datasets are presented in the main paper, additional supporting files or GenBank (http://www.ncbi.nlm.nih.gov/genbank/).

\section{Ethics approval and consent to participate}

Informed consent/assent was obtained from each participant. The study protocol was approved by institutional review boards at the Pennsylvania State

University, the Thai Ministry of Health, and Kunming Medical University, China.

\section{Funding}

This study was supported by a grant from the National Institute of Allergy and Infectious Diseases, National Institutes of Health (U19AI089672).

Received: 23 August 2016 Accepted: 7 November 2016

Published online: 15 November 2016

\section{References}

1. Singh B, Daneshvar C. Human infections and detection of Plasmodium knowlesi. Clin Microbiol Rev. 2013:26:165-84.

2. Gething PW, Elyazar IR, Moyes CL, Smith DL, Battle KE, Guerra CA, et al. A long neglected world malaria map: Plasmodium vivax endemicity in 2010. PLoS Negl Trop Dis. 2012;6:e1814.

3. Mueller I, Zimmerman PA, Reeder JC. Plasmodium malariae and Plasmodium ovale-the "bashful" malaria parasites. Trends Parasitol. 2007;23:278-83.

4. Collins WE, Jeffery GM. Plasmodium malariae: parasite and disease. Clin Microbiol Rev. 2007;20:579-92.

5. Collins WE, Jeffery GM. Plasmodium ovale: parasite and disease. Clin Microbiol Rev. 2005;18:570-81.

6. Trape JF, Rogier C, Konate L, Diagne N, Bouganali H, Canque B, et al. The Dielmo project: a longitudinal study of natural malaria infection and the mechanisms of protective immunity in a community living in a holoendemic area of Senegal. Am J Trop Med Hyg. 1994;51:123-37.

7. Koita OA, Sangare L, Sango HA, Dao S, Keita N, Maiga M, et al. Effect of seasonality and ecological factors on the prevalence of the four malaria parasite species in northern Mali. J Trop Med. 2012;2012:367160.

8. Roucher C, Rogier C, Sokhna C, Tall A, Trape JF. A 20-year longitudinal study of Plasmodium ovale and Plasmodium malariae prevalence and morbidity in a West African population. PLoS ONE. 2014:9:e87169.

9. Trape JF, Tall A, Sokhna C, Ly AB, Diagne N, Ndiath O, et al. The rise and fall of malaria in a West African rural community, Dielmo, Senegal, from 1990 to 2012: a 22 year longitudinal study. Lancet Infect Dis. 2014;14:476-88.

10. Doderer-Lang C, Atchade PS, Meckert L, Haar E, Perrotey S, Filisetti D, et al. The ears of the African elephant: unexpected high seroprevalence of Plasmodium ovale and Plasmodium malariae in healthy populations in Western Africa. Malar J. 2014;13:240.

11. Snounou G, Viriyakosol S, Jarra W, Thaithong S, Brown KN. Identification of the four human malaria parasite species in field samples by the polymerase chain reaction and detection of a high prevalence of mixed infections. Mol Biochem Parasitol. 1993:58:283-92.

12. Sutherland CJ, Tanomsing N, Nolder D, Oguike M, Jennison C, Pukrittayakamee $S$, et al. Two nonrecombining sympatric forms of the human malaria parasite Plasmodium ovale occur globally. J Infect Dis. 2010;201:1544-50.

13. Ansari HR, Templeton TJ, Subudhi AK, Ramaprasad A, Tang J, Lu F, et al. Genome-scale comparison of expanded gene families in Plasmodium ovale wallikeri and Plasmodium ovale curtisi with Plasmodium malariae and with other Plasmodium species. Int J Parasitol. 2016;46:685-96. 
14. Sitali L, Chipeta J, Miller JM, Moonga HB, Kumar N, Moss WJ, et al. Patterns of mixed Plasmodium species infections among children 6 years and under in selected malaria hyper-endemic communities of Zambia: population-based survey observations. BMC Infect Dis. 2015;15:204.

15. Getnet G, Getie S, Srivastava M, Birhan W, Fola AA, Noedl H. Diagnostic performance of rapid diagnostic tests for the diagnosis of malaria at public health facilities in Northwest Ethiopia. Trop Med Int Health. 2015;20:1564-8.

16. Zhou X, Huang JL, Njuabe MT, Li SG, Chen JH, Zhou XN. A molecular survey of febrile cases in malaria-endemic areas along China-Myanmar border in Yunnan province, People's Republic of China. Parasite. 2014;21:27.

17. Oguike MC, Betson M, Burke M, Nolder D, Stothard JR, Kleinschmidt I, et al. Plasmodium ovale curtisi and Plasmodium ovale wallikeri circulate simultaneously in African communities. Int J Parasitol. 2011;41:677-83.

18. Calderaro A, Piccolo G, Gorrini C, Rossi S, Montecchini S, Dell'Anna ML, et al. Accurate identification of the six human Plasmodium spp. causing imported malaria, including Plasmodium ovale wallikeri and Plasmodium knowlesi. Malar J. 2013;12:321.

19. Kawamoto F, Liu Q, Ferreira MU, Tantular IS. How prevalent are Plasmodium ovale and P. malariae in East Asia? Parasitol Today. 1999;15:422-6.

20. Eyles $\mathrm{DE}$, Wharton $\mathrm{RH}$, Cheong $\mathrm{WH}$, Warren $\mathrm{M}$. Studies on ùalaria and Anopheles balabacensis in Cambodia. Bull World Health Organ. 1964;30:7-21

21. Steenkeste $N$, Rogers WO, Okell L, Jeanne I, Incardona S, Duval L, et al. Sub-microscopic malaria cases and mixed malaria infection in a remote area of high malaria endemicity in Rattanakiri province, Cambodia: implication for malaria elimination. Malar J. 2010;9:108.

22. Sluydts V, Heng S, Coosemans M, Van Roey K, Gryseels C, Canier L, et al. Spatial clustering and risk factors of malaria infections in Ratanakiri Province, Cambodia. Malar J. 2014;13:387

23. Liu Q, Zhu S, Mizuno S, Kimura M, Liu P, Isomura S, et al. Sequence variation in the small-subunit rRNA gene of Plasmodium malariae and prevalence of isolates with the variant sequence in Sichuan, China. J Clin Microbiol. 1998;36:3378-81.

24. Kawamoto F, Win TT, Mizuno S, Lin K, Kyaw O, Tantulart IS, et al. Unusual Plasmodium malariae-like parasites in southeast Asia. J Parasitol. 2002;88:350-7.

25. Wang RB, Zhang J, Zhang QF. Malaria baseline survey in four special regions of northern Myanmar near China: a cross-sectional study. Malar J. 2014;13:302.

26. Zhou M, Liu Q, Wongsrichanalai C, Suwonkerd W, Panart K, Prajakwong $\mathrm{S}$, et al. High prevalence of Plasmodium malariae and Plasmodium ovale in malaria patients along the Thai-Myanmar border, as revealed by acridine orange staining and PCR-based diagnoses. Trop Med Int Health 1998;3:304-12.

27. Putaporntip C, Hongsrimuang T, Seethamchai S, Kobasa T, Limkittikul $\mathrm{K}$, Cui L, et al. Differential prevalence of Plasmodium infections and cryptic Plasmodium knowlesi malaria in humans in Thailand. J Infect Dis. 2009;199:1143-50.

28. Swan H, Sloan L, Muyombwe A, Chavalitshewinkoon-Petmitr P, Krudsood $\mathrm{S}$, Leowattana W, et al. Evaluation of a real-time polymerase chain reaction assay for the diagnosis of malaria in patients from Thailand. Am J Trop Med Hyg. 2005;73:850-4

29. Paxton LA, Slutsker L, Schultz LJ, Luby SP, Meriwether R, Matson P, et al. Imported malaria in Montagnard refugees settling in North Carolina: implications for prevention and control. Am J Trop Med Hyg. 1996;54:54-7.

30. Nguyen $\mathrm{HV}$, van den Eede $P$, van Overmeir $C$, Thang ND, le Hung $X$, D'Alessandro $U$, et al. Marked age-dependent prevalence of symptomatic and patent infections and complexity of distribution of human Plasmodium species in central Vietnam. Am J Trop Med Hyg. 2012;87:989-95.

31. Karnasuta C, Pongvongsa T, Jongsakul K, Na Nakorn A, Watt G. Plasmodium ovale in Lao PDR. Southeast Asian J Trop Med Public Health. 1997;28:746-7.

32. Toma H, Kobayashi J, Vannachone B, Arakawa T, Sato Y, Nambanya S, et al. Plasmodium ovale infections detected by PCR assay in Lao PDR. Southeast Asian J Trop Med Public Health. 1999;30:620-2.

33. Win TT, Lin K, Mizuno S, Zhou M, Liu Q, Ferreira MU, et al. Wide distribution of Plasmodium ovale in Myanmar. Trop Med Int Health. 2002;7:231-9.
34. Yan J, Li N, Wei X, Li P, Zhao Z, Wang L, et al. Performance of two rapid diagnostic tests for malaria diagnosis at the China-Myanmar border area. Malar J. 2013;12:73.

35. Kawamoto F, Miyake H, Kaneko O, Kimura M, Nguyen TD, Liu Q, et al. Sequence variation in the $18 \mathrm{~S}$ rRNA gene, a target for PCR-based malaria diagnosis, in Plasmodium ovale from southern Vietnam. J Clin Microbiol. 1996:34:2287-9.

36. Yao YT, Wu CC. On tghe peculiar morphology of the malaria parasite from a patient and the possibility of its being Plasmodium ovale. Chin Med J. 1941;60:178-83.

37. Li M, Xia Z, Yan H. New type of SSUrDNA sequence was detected from both Plasmodium ovale curtisi and Plasmodium ovale wallikeri samples. Malar J. 2014;13:216.

38. Zhang L, Feng J, Xia ZG. Malaria situation in the People's Republic of China in 2013 (in Chinese). Zhongguo Ji Sheng Chong Xue Yu Ji Sheng Chong Bing Za Zhi. 2014;32:407-13.

39. Cui L, Yan G, Sattabongkot J, Cao Y, Chen B, Chen X, et al. Malaria in the Greater Mekong Subregion: heterogeneity and complexity. Acta Trop. 2012;121:227-39.

40. Sturrock HJ, Roberts KW, Wegbreit J, Ohrt C, Gosling RD. Tackling imported malaria: an elimination endgame. Am J Trop Med Hyg. 2015:93:139-44.

41. Feng J, Xiao H, Xia Z, Zhang L, Xiao N. Analysis of malaria epidemiological characteristics in the People's Republic of China, 2004-2013. Am J Trop Med Hyg. 2015;93:293-9.

42. Li $P$, Zhao Z, Wang $Y$, Xing $H$, Parker DM, Yang Z, et al. Nested PCR detection of malaria directly using blood filter paper samples from epidemiological surveys. Malar J. 2014;13:175.

43. Tanomsing N, Mayxay M, Newton PN, Nosten F, Dolecek C, Hien TT, et al. Genetic variability of Plasmodium malariae dihydropteroate synthase (dhps) in four Asian countries. PLoS ONE. 2014;9:e93942.

44. Tanomsing N, Imwong M, Pukrittayakamee S, Chotivanich K, Looareesuwan S, Mayxay M, et al. Genetic analysis of the dihydrofolate reductasethymidylate synthase gene from geographically diverse isolates of Plasmodium malariae. Antimicrob Agents Chemother. 2007:51:3523-30.

45. Birkenmeyer L, Muerhoff AS, Dawson GJ, Desai SM. Isolation and characterization of the MSP1 genes from Plasmodium malariae and Plasmodium ovale. Am J Trop Med Hyg. 2010;82:996-1003.

46. Guimaraes LO, Wunderlich G, Alves JM, Bueno MG, Rohe F, Catao-Dias JL, et al. Merozoite surface protein-1 genetic diversity in Plasmodium malariae and Plasmodium brasilianum from Brazil. BMC Infect Dis. 2015;15:529.

47. Tamura K, Stecher G, Peterson D, Filipski A, Kumar S. MEGA6: molecular evolutionary genetics analysis version 6.0. Mol Biol Evol. 2013;30:2725-9.

48. Putaporntip C, Hughes AL, Jongwutiwes S. Low level of sequence diversity at merozoite surface protein-1 locus of Plasmodium ovale curtisi and P. ovale wallikeri from Thai isolates. PLoS ONE. 2013;8:e58962.

49. Cowman AF, Morry MJ, Biggs BA, Cross GA, Foote SJ. Amino acid changes linked to pyrimethamine resistance in the dihydrofolate reductase-thymidylate synthase gene of Plasmodium falciparum. Proc Natl Acad Sci USA. 1988:85:9109-13.

50. Sirawaraporn W, Sathitkul T, Sirawaraporn R, Yuthavong Y, Santi DV. Antifolate-resistant mutants of Plasmodium falciparum dihydrofolate reductase. Proc Natl Acad Sci USA. 1997:94:1124-9.

51. Khim N, Kim S, Bouchier C, Tichit M, Ariey F, Fandeur T, et al. Reduced impact of pyrimethamine drug pressure on Plasmodium malariae dihydrofolate reductase gene. Antimicrob Agents Chemother. 2012;56:863-8.

52. Chavatte JM, Tan SB, Snounou G, Lin RT. Molecular characterization of misidentified Plasmodium ovale imported cases in Singapore. Malar J. 2015;14:454.

53. Li N, Parker DM, Yang Z, Fan Q, Zhou G, Ai G, et al. Risk factors associated with slide positivity among febrile patients in a conflict zone of northeastern Myanmar along the China-Myanmar border. Malar J. 2013;12:361.

54. de Pecoulas PE, Tahar R, Ouatas T, Mazabraud A, Basco LK. Sequence variations in the Plasmodium vivax dihydrofolate reductase-thymidylate synthase gene and their relationship with pyrimethamine resistance. Mol Biochem Parasitol. 1998;92:265-73. 